\title{
Computing Popov and Hermite Forms of Polynomial Matrices
}

\author{
G. Villard \\ LMC-IMAG, 46, av. F. Viallet, F38031 Grenoble Cedex \\ Gilles.VillardQimag.fr
}

\begin{abstract}
For a polynomial matrix $P(x)$ of degree $d$ in $\mathcal{M}_{n, n}(K[x])$ where $K$ is a commutative field, a reduction to the Hermite normal form can be computed in $O^{-}(n d M(n)+M(n d))$ arithmetic operations if $M(n)$ is the time required to multiply two $n \times n$ matrices over $K$. Further, a reduction can be computed using $O\left(\log ^{\lambda+1}(n d)\right)$ parallel arithmetic steps and $O(L(n d))$ processors if the same processor bound holds with time $O\left(\log ^{\lambda}(n d)\right)$ for determining the lexicographically first maximal linearly independent subset of the set of the columns of an $n d \times n d$ matrix over $K$. These results are obtamed by applying in the matrix case, the techniques used in the scalar case of the gcd of polynomials.
\end{abstract}

\section{Introduction}

The problem of computing the greatest common divisor (gcd) of scalar polynomials in $K[x]$ ( $K$ is a commutative field) or of polynomial matrices in $\mathcal{M}_{m, n}(K[x])$ has attracted a lot of attention and has many applications in linear systems, control and realization theory, see [19] and references therein. The reverse approach has also been widely considered, that is the use of linear system theory and especially of the theory of realizations $[20,15]$ for the computation of scalar polynomial gcd's $[2,3]$. This has led to various computational results: giving either efficient numerical procedures as for instance developed in [24] or both sequential and parallel complexity results $[6,7]$.

From these latter points of view, the multivariable case (using the linear system terminology, as opposed to the scalar case), involving computations with polynomial matrices [26], is much less studied. Precisely, this paper aims at using standard results from linear systems theory to obtain new and better complexity bounds for the computation of the Hermite normal form via the polynomial matrix gcd. Our approach uses a generalization from the scalar case to the matrix case of certain algorithm for computing the gcd of polynomials. Let $P(x)$ be a matrix in $\mathcal{M}_{n, n}(K[x])$ of degree $d$. The degree of a polynomial matrux is defined as being the maximum of the degrees of its entries. To not excessive-

Permission to make digital/hard copies of all or part of this material for personal or classroom use is granted without fee provided that the copies are not made or distributed for profit or commercial advantage, the copyright notice, the title of the publication and its date appear, and notice is given that copyright is by permission of the ACM, Inc To copy otherwise, to republish, to post on servers or to redistribute to lists, requires specific permission and/or fee. ISSAC'96, Zurich, Switzerland; (C) 1996 ACM 0-89791-796-0/96/07.. .\$350 ly burden our presentation, we assume the determinant of $P(x)$ to be non identically zero. The general case should be quite easily derived. Throughout the paper we use right (column) or left (row) equivalence. A unimodular matrix in $\mathcal{M}_{n, n}(K[x])$ is an invertible matrix thus its determinant is a nonzero element of the ground field $K$. Two polynomial matrices are right (resp. left) equivalent if they differ by a right (resp. left) unimodular factor. In the same way, the different normal forms will be either obtained by right equivalence and be column forms or by left equivalence and be row forms. For each form we give a unique definition by column operations, the other one follows obviously.

There exists [28] a unique matrix $R_{H}(x)$ right equivalent to $P(x)$ and under (column) Hermite normal form:

$$
R_{H}(x)=P(x) V(x)
$$

with $V(x)$ unimodular in $\mathcal{M}_{n, n}(K[x])$. The Hermite form is an upper triangular form which diagonal entries are monic and such that in each row the entries following the diagonal entry are of lower degrees.

- Sequential point of view. Over an abstract field $K$ the Hermite form can be computed in sequential time $\tilde{O}\left(n^{4} d\right)[18$, 16] using a classical approach. To consider a unique parameter, let $\mu$ be such that $n=O(\mu)$ and $d=O(\mu)$. The previous complexity is $O^{-}\left(\mu^{5}\right)$. The notation $O^{-}$stands for a big "O" up to $\log$ terms, $O(n)$ is $O\left(n \log ^{\alpha} n\right)$ for some positive integer $\alpha$. Fast matrix multiplication techniques can be applied to improve the above cost. The problem of finding a unimodular triangularization $R_{T}(x)=P(x) W(x)$ can be solved in time $\tilde{O(n d M(n))}[16]$ if $M(n)$ is the complexity of matrix product (then the Hermite form is obtained by reducing off-diagonal entries). To compute the Hermite form, an algorithm has been discovered in the meantime for integer matrices [37]. It carries over directly for polynomial matrices and computes the form also in time $\tilde{O}(n d M(n))$. Since one can take $M(n)=n^{24}$, this bound is $O\left(\mu^{4.4}\right)$. Using standard polynomial and matrix multiplications $O\left(n^{5} d^{2}\right)=O\left(\mu^{7}\right)$ arithmetic operations are required for the elimination over polynomials in [18] or $O\left(n^{4} d^{3}\right)=O\left(\mu^{7}\right)$ for the elimination over constants in $[8,27]$. The best Las Vegas probabilistic solution has been given in [36] for a unimodular triangularization only, its cost is $O\left(n^{4} d^{2}\right)=O\left(\mu^{6}\right)$.

Our method will compute the Hermite form in deterministic sequential time $\sigma^{-}(n d M(n)+M(n d))$ with fast arithmetic. Using standard arithmetic it will require $O\left(\mu^{6}\right)$ operations and thus match the cost of the best above randomized solution. Beyond a good theoretical complexity, especially in parallel, the new algorithm should thus provide very fast 
practical implementations in sequential.

At this point, we may emphasize the two key ideas we are going to exploit. On the one hand, instead of directly computing the Hermite form of $P(x)$, it will be deduced from an intermediate form that is widely used in linear system theory - the Popov form $[32,19]$. On the other hand, the computation of this latter form for $P(x)$ will be reduced to the computation of a well chosen matrix polynomial $\mathrm{gcd}$. This latter problem will be solved using block Hankel matrices (being inspired by the scalar case)

- Parallel point of view. Very few algorithms exist that compute the Hermite form fast using polynomially many processors. The solutions in $[18,16]$ are elimination processes and are thus highly sequential. The problem has been shown to belong to the class $\mathcal{N C}$ in [21]. Nevertheless, this latter approach involves $O\left(n^{2} d\right)$ structured linear systems of dimension $n^{3} d$. Since quite prohibitive, the cost has not been precisely computed by the authors. For a number of parallel steps in $O\left(\log ^{3} \mu\right)$, one can evaluate the number of needed processors to be in $O\left(\mu^{11}\right)$ over fields of characteristic zero and in $O\left(\mu^{18}\right)$ over any fields. Another solution could be to compute the form by obtaining (in parallel) a row echelon form of a generalized Sylvester matrix - Toeplitz-like - as done in $[8,27]$ (in sequential). For a time $O\left(\log ^{3} \mu\right)$, one can also evaluate the number of processors (this solution has not been investigated) to be in $O\left(\mu^{9}\right)$ over fields of characteristic zero and in $O\left(\mu^{16.5}\right)$ over any fields. To the knowledge of the author, no better deterministic bound is available.

The parallel version of our algorithm will substantially decrease these costs. An implementation will be possible in time $O\left(\log ^{4}(n d)\right)$ using $O\left(n^{3 / 2} d M(n)+(n d)^{1 / 2} M(n d)\right)$ or $O\left(\mu^{5.8}\right)$ processors over fields of characteristic zero. Over any field the new bound is $O^{-}\left((n d)^{4} M(n d)\right)$ or $O\left(\mu^{12.8}\right)$. The processor inefficiency of the algorithm, defined as the ratio of the parallel work over the sequential complexity is $O\left((n d)^{1 / 2}\right)=O(\mu)$ for fields of characteristic zero and $O\left(\mu^{8}\right)$ in the general case. These values essentially come from the inefficiency of known solutions [7] to the problem of computing the nullspace of a matrix, or more precisely, as we will see, to the problem of determining the lexicographically first maximal linearly independent subset of the set of the column$\mathrm{s}$ of a matrix. However, processor-efficient algorithms of Las Vegas type are available for these latter problems $[22,23,12]$. We will see that they allow to compute the Hermite form, using $\tilde{O}(n d M(n)+M(n d))=O\left(\mu^{48}\right)$ processors, in time $O\left(\log ^{4}(n d)\right)$ if $K$ is of characteristic zero or of characteristic $p$ greater than $n d$ and otherwise in time $O\left(\log ^{5}(n d) / \log p\right)$.

These complexities do not express the boolean computational cost of the algorithms. We will not detail the corresponding studies in this paper. Further developments should be done [35] using the Bezoutian for matrix polynomial$\mathrm{s}[4,34]$. From results in [7] concerning the scalar polynomial gcd, very good bounds may be expected.

The paper is organized as follows: we begin with some results on the polynomial matrix gcd in $\S 1$ and on the minimal realization problem in $\S 2$. Then we will see in $\S 3$ how these suitable results lead to the algorithm for computing the Popov form. This is the first step toward the Hermite form. The Popov form is read-off the nullspace of a block Hankel matrix which entries are computed from the input matrix $P(x)$. Based on this result, we then devise a sequential algorithm and a parallel algorithm in $\$ 4$ for computing the Hermite normal form of an invertible matrix from the previously computed Popov form. The cost analysis will be done in $\S 5$. The reader may directly refer to $\S 3$ for a sketch of algorithm 1 which computes the Popov form and to $\$ 4$ for a sketch of algorithm 2 which computes the Hermite form from the former one.

\section{Polynomial matrix ged}

A greatest common right divisor (gcrd) of two polynomial matrices can be defined provided they have the same number of columns. In the following we will restrict ourselves to the case where $N(x)$ is in $\mathcal{M}_{m, n}(K[x])$ and $D(x)$ is nonsingular in $\mathcal{M}_{n, n}(K[x])$.

\subsection{Definition}

A gcrd of $N(x)$ and $D(x)$ is (see [28]) any polynomial matrix $G(x)$ such that I) $G(x)$ is a right divisor of $N(x)$ and $D(x)$ : for some matrices $\bar{N}(x)$ and $\bar{D}(x)$ we have

$$
N(x)=\bar{N}(x) G(x), \quad D(x)=\bar{D}(x) G(x) ;
$$

II) any other right divisor $G_{1}(x)$ of $N(x)$ and $D(x)$ is a right divisor of $G(x): G(x)=T(x) G_{1}(x)$ for some polynomial matrix $T(x)$. Gcrd's are not unique, but if ${ }^{t}\left[{ }^{t} N(x),{ }^{t} D(x)\right]$ is of rank $n$-here this is true by assumption - then all gcrd's of $N(x)$ and $D(x)$ must be nonsingular in $\mathcal{M}_{n, n}(K[x])$ and left equivalent. Here and throughout the text, for a matrix $M,{ }^{t} M$ denotes its transpose. In order to define the gcrd uniquely, it is thus sufficient to consider any row normal form to represent the equivalence class of all the gcrd's. The row Hermite form is a possible choice; besides, one way to compute a gcrd [28] is to compute the Hermite form $L_{H}(x)$ of ${ }^{t}\left[{ }^{t} N(x),{ }^{t} D(x)\right]$ by left equivalence:

$$
U(x)\left[\begin{array}{l}
N(x) \\
D(x)
\end{array}\right]=\left[\begin{array}{c}
G(x) \\
0
\end{array}\right]
$$

And $G(x)$ is a gcrd of $N(x)$ and $D(x)$. But precisely, to devise efficient algorithms for the Hermite form, we are going to adopt the converse approach, first computing a gcrd and then obtaining the form itself.

\subsection{The Popov form}

Another choice to represent the gcrd's is to use the Popov form. This will be more judicious because the highest degree of the entries of the Popov form of a matrix, will be no greater than the degree of the matrix. This property is clearly false for the Hermite form. We define the column Popov form. For $G(x)$ in $\mathcal{M}_{n, n}(K[x])$ let $d_{j}, 1 \leq j \leq n$, the $j$-th column degree, be the degree of the $j$-th column of $G(x)$. The coefficient vector of $x^{d_{j}}$ is the $j$-th leading column coefficient vector. We let $[G(x)]_{c}$ be the matrix of these leading vectors. A matrix $G(x)$ is said to be column reduced (or column proper in [41]) if rank $[G(x)]_{c}=$ rank $G(x)$, it satisfies: $\operatorname{deg} \operatorname{det} G(x)=\sum_{j=1}^{n} d_{j}$. If, in addition, $G(x)$ satisfies the following properties, we shall say that $G(x)$ is in Popov form: I) the column degrees are increasingly ordered; II) the last entry of degree $d_{j}$ in each column is monic, it is called the pivot of column $j$ with row index $r_{j}$; III) if $d_{\jmath}=d_{k}$ and $j<k$ then $r_{\jmath}<r_{k}$; IV) all entries in a row containing a pivot element have degrees lower than that of the pivot element. The Popov form is normal and satisfies a degree property:

Theorem 1 ([32, 19]) Any two right (resp. left) equivalent matrices in $\mathcal{M}_{n, n}(K[x])$ of degree $d_{1}$ and $d_{2}$ have the same column (resp. row) Popov form of degree at most $\min \left\{d_{1}, d_{2}\right\}$. 
Example 1. The following matrix $P(x)$ is not column reduced, rank $[P(x)]_{c}=1$.

$$
\left[\begin{array}{cc}
-2 x-2+x^{3} & 4 x+5-x^{3} \\
-x^{2}+2+4 x & 2 x^{2}-5-4 x
\end{array}\right] .
$$

The next one is right equivalent to $P(x)$ and is column reduced but not under Popov form since it does not obey requirement IV):

$$
\left[\begin{array}{cc}
2 x+3 & 4 x+5-x^{3} \\
x^{2}-3 & 2 x^{2}-5-4 x
\end{array}\right]
$$

The last matrix is also right equivalent to $P(x)$, now it is under Popov form:

$$
\left[\begin{array}{cc}
2 x+3 & 1+x^{3} \\
x^{2}-3 & -1+4 x
\end{array}\right]
$$

with $d_{1}=2, d_{2}=3$ and $r_{1}=2, r_{2}=1$.

We may point out some related works. The univariate determinant computation in [29] may be viewed as the computation of a column reduced form. More generally, strong links are well known between column reduced forms and the determination of the finite eigenstructure of a polynomial matrix $[39,38]$ and also with the factorization algorithm of Kublanovskaya [5]. Nevertheless, we are not aware of any study of the number of operations needed to compute the Popov form. From the methods in [32, 19] or in [5] and by analogy with results in [38] one can see that $O\left(n d^{2} M(n)\right)=O\left(\mu^{5.4}\right)$ is a correct bound. Even if of main theoretical importance such a form has poor properties from a numerical analysis point of view.

As said previously the Popov form will be the first step toward the Hermite form. It will be computed in $\S 3$ by a polynomial matrix gcrd operation. Apart from using matrix equivalence and identity (1), a gcrd can be computed from the generalized Sylvester resultant matrix in [8]. However this method seems to lead to the same conclusions than when using the Sylvester matrix in [27] and does not seem fully appropriate to parallelism. Indeed the two methods differ only by the form of the gcrd that is computed (Hermite versus Popov form) and suffer from the fact that the degree of the associated transformation matrix can be in $O(n d)$, even though the degree of the input matrix and of the Popov form of the gcrd are in $O(d)$. A last method for polynomial gcrd is to compute an irreducible fraction. The right matrix fraction description

$$
H(x)=N(x) D^{-1}(x)
$$

is irreducible if $N(x)$ and $D(x)$ are right coprime or, equivalently, if any gcrd of $N(x)$ and $D(x)$ is a unimodular matrix. The same can be defined in a dual manner on the left with $H(x)=C^{-1}(x) L(x)$. If a right matrix fraction description is not irreducible, suppose we have a way to compute an irreducible description $\bar{N}(x) \bar{D}^{-1}(x)$ of $H(x)$, then a gcrd of $N(x)$ and $D(x)$ is computed as $G(x)=\bar{D}^{-1}(x) D(x)[8]$. This is basically the approach used for the scalar polynomial gcd in $[6,7]$. We will see in next section that the same can be done for matrices, and how we can compute an irreducible fraction.

\section{The minimal realization problem}

From a system-theoretical point of view, normal forms appear from the general minimal (or partial) realization problem $[20,15]$.

\subsection{The problem}

Consider $H(x)=N(x) D^{-1}(x)$ a strictly proper rational matrix:

Definition $1 A$ fraction $u(x) / v(x)$ is structly proper if deg $u<$ deg $v$. A matrix fraction in $\mathcal{M}_{m, n}(K(x))$ is strictly proper if all its entries are strictly proper.

Thus $H(x)$ has a formal expansion at infinity

$$
H(x)=\Sigma_{\imath=1}^{\infty} H_{\imath} x^{-i}
$$

where the $H_{2}$ 's are matrices in $\mathcal{M}_{m, n}(K)$. A triplet $\Sigma=$ $(C, A, B)$ of matrices in $\mathcal{M}_{m, \nu}(K), \mathcal{M}_{\nu, \nu}(K)$ and $\mathcal{M}_{\nu, n}(K)$ respectively such that:

$$
C A^{\imath-1} B=H_{\imath} \text { for } i=1, \ldots
$$

is called a realization of $H(x)$. Moreover $\Sigma$ satisfies:

$$
H(x)=C(x I-A)^{-1} B .
$$

The realization is called minimal if the dimension $\nu$ is minimal, one says that $H(x)$ as transfer function have minimal realization $\Sigma$. Given $H(x)$, the problem is to find such a minimal realization. The $H_{i}$ 's are called the Markov parameters. In the scalar case $(n, m=1)$, for a transfer function $h(x)=u(x) / v(x)$, a minimal realization gives a new representation of $h(x)$ as the quotient of two relatively prime polynomials: $h(x)=\bar{u}(x) / \bar{v}(x)$. So the reduction of the problem of computing the gcd of two polynomials $u(x)$ and $v(x)$ to the minimal realization problem is obvious. From $u(x)$ and $v(x)$ compute a minimal realization of the $h_{\imath}$ 's, this realization gives $\bar{u}(x)$ and $\bar{v}(x)$ and consequently the gcd. We refer to $[6,7]$ for corresponding algorithms.

In the multivariable case (using the linear system terminology), for a transfer matrix given as a matrix fraction $H(x)=N(x) D^{-1}(x)$, a minimal realization of $H(x)$ provides an irreducible fraction $\bar{N}(x) \bar{D}^{-1}(x)$ of $H(x)$ with $\bar{D}(x)$ under special form $[26,19]$. Thus the polynomial matrix gcd problem is also reduced to the minimal realization problem.

\subsection{A minimal realization from the Markov parameters}

For $H(x)=N(x) D^{-1}(x)$ strictly proper rational, we now look at the problem of finding a minimal realization matching the corresponding set of Markov parameters $H_{2}, i>1$, given by (3) and (4). The problem can be solved by finding two right coprime matrices $\bar{N}(x)$ and $\bar{D}(x)$ such that

$$
H(x)=N(x) D^{-1}(x)=\bar{N}(x) \bar{D}^{-1}(x)=\Sigma_{i=1}^{\infty} H_{2} x^{-\imath}
$$

and such that $\bar{D}(x)$ is column reduced.

Notation 1. For any integer $k \geq 1$, the $H_{2}$ 's define the block Hankel $k m \times k n$ matrix $M(k)$ whose $(i, j)$ block is $H_{n+j-1}$.

From (6), the special structures of the denominator matrix $\bar{D}(x)$ are going to be reflected in special relations between the columns of $M(\nu+1)$.

Propositions below are very useful facts derived from [26, 19]. They will directly lead, at next section, to a procedure for fraction reduction. Our scheme of proof is inspired from the scalar case in $[6,7]$. We give two propositions. Their two assertions correspond to the "if" and the "only if" part respectively of the following theorem the proof of which is omitted. 
Theorem 2 (theorem 6.5-1, [19]). A matrix fraction description is irreducible if and only if the determinantal degree of the denominator matrix is the dimension of any minimal realization of the fraction (right and left irreducible descriptions of the same fraction have the same denominator determinantal degree).

The following result is derived from [26, 19]. We state it by emphasizing the degree of the denominator matrix of the reduced fraction, this will be a relevant parameter to obtain good complexity bounds. The determinantal degree of the matrices plays the role of the polynomial degree during the euclidean division in the scalar case.

Proposition 1 Let $H(x)=N(x) D^{-1}(x)$ be strictly proper in $\mathcal{M}_{m, n}(K(x))$ with $D(x)$ in $\mathcal{M}_{n, n}(K[x])$. Let $\nu$ be the dimension of a minimal realization of $H(x)$. If $H(x)$ has a right irreducible description $\bar{N}(x) \bar{D}^{-1}(x)$ and a left irreducible description $\bar{C}^{-1}(x) \bar{L}(x)$ such that the denominator matrices $\bar{D}(x)$ and $\bar{C}(x)$ have degrees bounded by $\delta$, then $\operatorname{rank} M(\delta)=\nu$ (notation 1$)$.

Proof (Compare proposition 2.1 in [6] for the scalar polynomial gcd). By minimality of $\nu$ we know that rank $M(\nu+i)=$ rank $M(\infty)=\nu, i \geq 0$ ([43] or lemma 6.5-7, [19]). We apply the "if" part of theorem 2. Since $\bar{N}(x) \bar{D}^{-1}(x)$ is irreducible, the determinantal degree of $\bar{D}(x)$ is $\nu$. Using theorem $1, \bar{D}(x)$ can be brought in Popov form $T(x)$ up to a right unimodular factor: $\bar{D}(x) V(x)=T(x)$. The degree of the entries of $T(x)$ are also bounded by $\delta$ the degree of $\bar{D}(x)$. We denote by $T_{i}$ the $i$-th coefficient matrix of $T(x)$. Let $S(x)=\bar{N}(x) V(x)$, by (6), $S(x) T^{-1}(x)=\Sigma_{\imath=1}^{\infty} H_{2} x^{-i}$ or $\left(H_{1} x^{-1}+H_{2} x^{-2}+\ldots\right)\left(T_{0}+T_{1} x+\ldots T_{\delta} x^{\delta}\right)=S(x)$. Since $S(x)$ is a matrix polynomial, comparing the coefficient of $x^{-\imath}, i \geq 1$, on both sides of the equation we obtain:

$$
H_{\imath} T_{0}+H_{\imath+1} T_{1}+\ldots+H_{i+\delta} T_{\delta}=0, \quad i \geq 1 .
$$

Thus there is a strong relationship between the entries of $T(x)$ and the dependency relations between the columns of $M(\nu+1)$. Indeed, let $T(x)=\left\{\Sigma_{k=0}^{\delta} t_{l j k} x^{k}\right\}$. From the definition of the Popov form, let $d_{j}$ be the $j$-th column degree of $T(x)$ and let $r_{j}$ be the index of the corresponding pivot entry. Identity (7) for $i \geq 1$ and for the $j$-th column of $T(x)$ gives:

$$
M_{r_{j}}^{\left(d_{j}+1\right)}=\Sigma_{l=1}^{r_{j}-1} M_{l}^{\left(d_{j}+1\right)} t_{l j d_{j}}+\Sigma_{l=1}^{n} \Sigma_{k=0}^{d_{j}-1} M_{l}^{(k+1)} t_{l j k}
$$

for $1 \leq j \leq n$ and with $M_{l}^{(k)}$ denoting the column $l$ of the $k$-th block column of $M(\nu+1)$. For any $l$, only the columns $M_{r_{l}}^{(1)}, \ldots, M_{r_{l}}^{\left(d_{j}+1\right)}$ can appear in the right hand side of (8); the fact that $T(x)$ is in Popov form (requirement IV) implies that only the columns $M_{r_{l}}^{(1)}, \ldots, M_{r_{l}}^{\left(d_{l}\right)}$ can appear. So, if we look at all the identities (8) when $j$ varies, $1 \leq j \leq n$, for each $r_{\jmath}$, only the columns $M_{r_{j}}^{(1)}, \ldots, M_{r_{\jmath}}^{\left(d_{\jmath}\right)}$ can appear in some right hand side. By assumption there are $\Sigma_{1}^{n} d_{j}=\nu$ such columns. We denote $\bar{M}_{\delta}$ the submatrix of $M(\delta)$ built from these $\nu$ columns taken in the order they appear in $M(\delta)$. If any column degree is zero, say the $j_{0}$-th, then the columns $\bar{M}_{j_{0}}^{(k)}, k \geq 1$, are not involved in $\bar{M}_{\delta}$. We denote by $\rho, 1 \leq \rho \leq n$, the number of nonzero column degrees. If $\pi$ is an index permutation such that the $r_{\pi(j)}$ are increasingly ordered, $d_{\pi(\jmath)} \neq 0$ for $1 \leq j \leq \rho$ and $d_{\pi(j)}=0$ for $j \geq \rho+1$,

$$
\bar{M}_{\delta}=\left[\bar{M}_{r_{\pi(1)}}^{(1)}, \ldots, \bar{M}_{r_{\pi(\rho)}}^{(1)}, \ldots\right] \in \mathcal{M}_{\delta m, \nu}
$$

with $\bar{M}_{l}^{(k)}$ denoting the column $l$ of the $k$-th block column of $M(\delta)$. With this choice of columns in $M(\delta)$, it is natural to consider the corresponding $\nu$ rows of ${ }^{t}\left[T_{0}^{t},{ }^{t} T_{1}, \ldots,{ }^{t} T_{\delta}\right]$ as a $\nu \times n$ matrix:

$$
{ }^{t}\left[\bar{T}_{0},{ }^{t} \bar{T}_{1}, \ldots,{ }^{t} \bar{T}_{\delta-1}\right]
$$

and the rows $d_{\pi(1)}+1, \ldots, d_{\pi(n)}+1$ form $\bar{T}_{\delta}$. The matrix $\bar{T}_{\delta}$ is a $n \times n$ permutation matrix since it is a submatrix of $[T(x)]_{c}$ and since the pivot entries in $T(x)$ are monic. Now, we may rewrite relations (7) and (8) in matrix form:

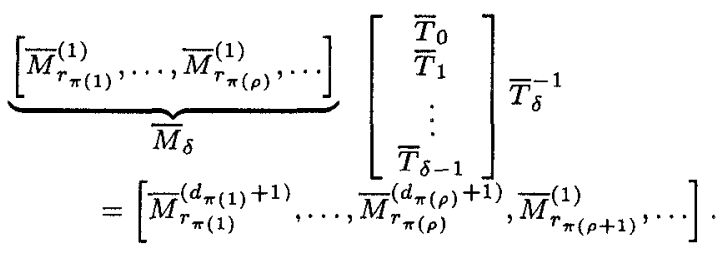

We are going to show that $\bar{M}_{\delta}$ (and thus $M(\delta)$ ) is of rank $\nu$. By contradiction, if it is not, we show that the fractions $\bar{N}(x) \bar{D}^{-1}(x)$ and $\bar{C}^{-1}(x) \bar{L}(x)$ was not irreducible. Indeed, if $\bar{M}_{\delta}$ has rank strictly lower than $\nu$, the linear system (9) gives other solutions than $T(x)$. One such solution, $T^{\prime}(x)$, can be constructed to have (at least) one column degree strictly lower than the corresponding column degree of $T(x)$, the others being lower or equal. Thus $T^{\prime}(x)$ has determinantal degree strictly lower than $\nu$. We also construct the associated numerator $S^{\prime}(x)$ using $[19,7]$ :

$$
\left[\begin{array}{c}
S_{\delta-1}^{\prime} \\
\vdots \\
S_{0}^{\prime}
\end{array}\right]=\left[\begin{array}{cccc}
H_{1} & 0 & \ldots & 0 \\
H_{2} & H_{1} & \ddots & \vdots \\
\vdots & \ddots & \ddots & 0 \\
H_{\delta} & \ldots & \ldots & H_{1}
\end{array}\right]\left[\begin{array}{c}
T_{\delta}^{\prime} \\
\vdots \\
T_{1}^{\prime}
\end{array}\right]
$$

Now, the expansions of $H(x)$ using $\bar{C}^{-1}(x) \bar{L}(x)$ and using $S^{\prime}(x)\left(T^{\prime}(x)\right)^{-1}$ at infinity coincide up to the order $2 \delta$ :

$$
\bar{C}^{-1}\left(x^{-1}\right) \bar{L}\left(x^{-1}\right) / x-S^{\prime}\left(x^{-1}\right)\left(T^{\prime}\left(x^{-1}\right)\right)^{-1} / x \equiv 0 \bmod x^{2 \delta \delta}
$$

then there exists a polynomial matrix $Q(x)$ such that

$\bar{L}\left(x^{-1}\right) T^{\prime}\left(x^{-1}\right)-\bar{C}\left(x^{-1}\right) S^{\prime}\left(x^{-1}\right)=x^{2 \delta+1} \bar{C}\left(x^{-1}\right) Q(x) T^{\prime}\left(x^{-1}\right)$.

But $\bar{C}(x)$ and $T^{\prime}(x)$ have degrees lower than $\delta$, that is

$$
\bar{L}\left(x^{-1}\right) T^{\prime}\left(x^{-1}\right)-\bar{C}\left(x^{-1}\right) S^{\prime}\left(x^{-1}\right) \equiv 0 \bmod x
$$

and

$$
\bar{L}(x) T^{\prime}(x)-\bar{C}(x) S^{\prime}(x)=0 .
$$

Now, since $\bar{C}^{-1}(x) \bar{L}(x)$ is irreducible, the determinantal degree of $\bar{C}(x)$ must be (lemma 6.3-8, [19]) at most equal to the one of $T^{\prime}(x)$ and thus strictly lower than $\nu$. This contradicts the irreducibility of the fractions.

Proposition 2 Let $H(x)=N(x) D^{-1}(x)$ be as in proposition 1. If $\bar{D}(x)=D_{\delta} x^{\delta}+\ldots+D_{0}$ in $\mathcal{M}_{n, n}(K[x])$ is of determinantal degree $\nu$ and such that

$$
\underbrace{\left[\begin{array}{cccc}
H_{1} & H_{2} & \ldots & H_{\delta+1} \\
H_{2} & H_{3} & \ldots & H_{\delta+2} \\
\vdots & & & \vdots \\
H_{\delta+1} & \ldots & \ldots & H_{2 \delta+1}
\end{array}\right]}_{M(\delta+1)}\left[\begin{array}{c}
D_{0} \\
D_{1} \\
\vdots \\
D_{\delta}
\end{array}\right]=\left[\begin{array}{c}
0 \\
0 \\
\vdots \\
0
\end{array}\right]
$$


then $D(x)=\bar{D}(x) G(x)$ where $G(x)$ us a gcrd of $N(x)$ and $D(x)$.

Proof (Compare proposition 3.6 in [6] for the scalar polynomial gcd). This is the "only if" part of theorem 2 and corresponds to the method proposed in [26] to compute a minimal realization. Indeed, once $\bar{D}(x)$ in Popov form is computed, a satisfying triplet $(C, A, B)$ can be readily obtained $[32,19]$. If $\bar{D}(x)$ is nonsingular and satisfies (11) then one can built a description $\bar{N}(x) \bar{D}^{-1}(x)$ of $H(x)$ (using arguments similar to those used in the proof of proposition 1). And since the determinantal degree of the denominator is $\nu$ we know that the fraction is irreducible. Using for instance lemma $6.5-5$ in [19], we get that $\bar{D}(x)=D(x) G^{-1}(x)$ where $G(x)$ is a gcrd of $N(x)$ and $D(x)$ (the fraction has been "simplified by" $G(x))$.

In the rest of the paper, we will view the above propositions as giving a procedure to reduce a matrix fraction (or, equivalently, to compute a gcrd).

\subsection{Reduction of a matrix fraction}

We now relate the fraction reduction to the problem $\mathcal{L}(n)$ of finding the lexicographically first maximal linearly independent subset of the set of the columns of a matrix in $\mathcal{M}_{n, n}(K)$. Here, the problem is applied to a block Hankel matrix. We may (reasonably) assume that solving this problem is more expensive than matrix product or inversion.

Lemma 1 Let $H(x)=N(x) D^{-1}(x)$ be in $\mathcal{M}_{m, n}(K(x))$, $m \leq n$, be as in proposition 1, in partıcular there exist left and right irreducible fraction descriptions of $H(x)$ whose denominator matrices have degrees bounded by $\delta$. A reduced fraction description $H(x)=\bar{N}(x) \bar{D}^{-1}(x)$ with $\bar{D}(x)$ under Popov form can be computed in two solutions of the problem $\mathcal{L}(n \delta)$ for a block Hankel matrix with $m \times n$ blocks.

Proof. We first compute the matrix $M(\delta+1)$. This is done by computing for each entry $h_{i, j}(x)$ of $H(x)$ the Taylor expansion of $h_{\imath, \jmath}\left(x^{-1}\right) / x$ (this is clearly of lower cost than the rest). Now, from the linear system (11) we compute the linear system (9). Applying $\mathcal{L}(n \delta)$ to $M(\delta+1)$ gives by construction the submatrix $\bar{M}_{\delta}$. Since we choose the lexicographically first set of columns, the corresponding dependencies give a column reduced matrix $T(x)$ that is under Popov form up to some column permutations. This matrix is obtained by solving the system (9), for instance by isolating a square invertible submatrix of $\bar{M}_{\delta}$ using a second solution of $\mathcal{L}(n \delta)$ as done in [9] for the nullspace computation. Then $\bar{D}(x)$ is computed under Popov form simply by reordering the columns of $T(x)$ with respect to their degrees, and $\bar{N}(x)$ is deduced from identity (10). By construction, $\bar{D}(x)$ is of determinantal degree $\nu$, proposition 2 tells us that $\bar{N}(x) \bar{D}^{-1}(x)$ is irreducible.

Costs will be detailed in $\S 5$. Before concluding, let us point out well known links with the extended Euclidean algorithm (or Berlekamp-Massey algorithm) used in [10] to solve sparse linear equations. The corresponding problem and algorithm to find a generator of a matrix sequence correspond to the solutions of linear systems (11) and (9). Given the sequence of the $H_{2}$ 's, the minimum generator computed there, may be viewed as one of the column vectors of the denominator matrix $\bar{D}(x)$ of a well chosen transfer function.

\section{Computing the Popov form}

Applying above results, we now compute the column Popov form $T(x)$ of a matrix $P(x)$.

\subsection{Direct approach}

Since Popov forms appear at the denominator of irreducible fractions, the problem is to find a suitable transfer function $H(x)$ associated to $P(x)$ i.e. such that the Popov form of the denominator of an irreducible description of $H(x)$ is $T(x)$. But, as emphasized in [14], to study a nonsingular matrix polynomial $P(x)$ it is natural to study systems for which the transfer function is $H^{*}(x)=P^{-1}(x)$. Precisely we are going to see that $H^{*}(x)$ leads to a suitable choice $H(x)$ to compute $T(x)$. We denote by $\mathcal{I}(n, d)$ the problem of inverting a matrix of degree $d$ in $\mathcal{M}_{n, n}(K[x])$.

Algorithm 1. Computing the column Popov form.

The Popov form of $P(x)$ is computed by reducing the fraction $H(x)=\left(P^{*}(x) \bmod \Delta(x)\right) \Delta^{-1}(x)$ (lemma 2). The reduced fraction is found by solving a block Hankel system (lemma 1).

Input: a polynomial matrix $P(x)$.

$P^{*}(x) \leftarrow$ the adjoint of $P(x)$.

$\Delta(x) \leftarrow \operatorname{diag}(\operatorname{det} P(x), \ldots, \operatorname{det} P(x))$.

$N(x) \leftarrow P^{*}(x) \bmod \Delta(x)$.

Reduce $H(x)=N(x) \Delta^{-1}(x)$ applying lemma 1 :

Compute the expansion $H(x)=\Sigma H_{2} x^{-\imath}$.

Build the block Hankel matrix $M(\delta+1)$ as in (11).

$\bar{M}(\delta) \leftarrow$ the first independent columns of $M(\delta+1)$.

$\mathcal{T} \leftarrow$ the solution of the linear system $(9)$.

Build $T(x)$ from the entries of $\mathcal{T}$.

Output: the column Popov form $T(x)$ of $P(x)$.

Lemma 2 The column Popov form of a nonsingular matrix $P(x)$ of degree $d$ in $\mathcal{M}_{n, n}(K[x])$ can be computed in one matrix inversion $\mathcal{I}(n, d)$ and two computations of independent columns $\mathcal{L}(n d)$ of a block Hankel matrix.

Proof. Consider $H^{*}(x)=P^{-1}(x)$. If we denote by $P^{*}(x)$ the adjoint matrix of $P(x)$ and $\Delta(x)$ the diagonal matrix of dimension $n$ whose nonzero entries are the determinant of $P(x)$ :

$$
H^{*}(x)=P^{-1}(x)=P^{*}(x) \Delta^{-1}(x) .
$$

In the general case, $H^{*}(x)$ is not strictly proper. However, since we are going to focus on the reduced fraction, we can take:

$$
H(x)=N(x) \Delta^{-1}(x)=\left(P^{*}(x) \bmod \Delta(x)\right) \Delta^{-1}(x)
$$

with the obvious definition of the modulo $\Delta(x)$. This new transfer function is strictly proper and $G(x)$ is a gcrd of $P^{*}(x)$ and $\Delta(x)$ if and only if it is a gcrd of $N(x)$ and $\Delta(x)$. Now, $P^{*}(x)$ is such a gcrd thus every gcrd $G(x)$ of $N(x)$ and $\Delta(x)$ is given by

$$
G(x)=U(x) P^{*}(x)
$$

with $U(x)$ unimodular. Furthermore, any irreducible right fraction description $\bar{N}(x) \bar{D}^{-1}(x)$ of $H(x)$, obtained by "extracting" a gcrd from $N(x)$ and $\Delta(x)$ satisfies

$\bar{D}(x)=\Delta(x) G^{-1}(x)=\Delta(x)\left(P^{*}(x)\right)^{-1} U^{-1}(x)=P(x) V(x)$

with $V(x)$ unimodular. From the uniqueness of the Popov form, to compute the Popov form $T(x)$ of $P(x)$ thus reduces 
to compute an irreducible fraction description of $H(x)$ with denominator $\bar{D}(x)$ under Popov form: $T(x)=\bar{D}(x)$. Since the column and the row Popov forms of $P(x)$ are at most of degree $d$, there exist irreducible right and left fraction descriptions of $H(x)$ with denominators of degrees at most $d$, we conclude by applying lemma 1 to $H(x)$. One additional matrix inversion is needed to build $M(d+1)$.

\subsection{Dual approach}

Clearly, it is also possible to exactly match the scalar case for the polynomial gcd [6]. For $u(x)$ and $v(x)$, the authors of this paper proceed in two steps: first computing a reduced fraction $\bar{v}(x) / \bar{u}(x)$ then obtaining the gcd as $u(x) / \bar{u}(x)$. In the same way, we may consider $H(x)=P(x) \Delta^{-1}(x)$. The reduction of the fraction now gives as denominator the Popov form $T^{*}(x)$ of the adjoint matrix of $P(x), T(x)$ is easily deduced (row Popov form with this dual approach). This method compares favourably with the above one in the sense that it can be easily built for singular matrices $(\Delta(x)$ can be build from a well chosen nonzero minor of $P(x)$ ) [35]. But its main drawback is that the degree of $T^{*}(x)$ can be as large as $n d$. Thus one has to take $\delta=n d$ and to deal with $M(\delta)=M(n d)$ which is of dimensions $m n d \times n^{2} d$.

This dual point of view, when applied to the computation of the gcd of $n$ polynomials, resembles the method in [24] for this latter problem which is a particular case of the Popov or of the Hermite normal form for a $1 \times n$ row matrix. Adopting the system theoretic language, $M(\delta+1)$ in proposition 2 has to be viewed as a controllability matrix of an implicitly defined linear system $S(A, B)$. In the direct approach (resp. the dual approach), the controllable modes (the uncontrollable modes) of $S(A, B)$ provide the Popov form.

\section{Computing the Hermite form}

By lemma 2 it remains to focus on the computation of the column Hermite normal form $R_{H}(x)$ of $P(x)$ from the Popov one $T(x)$. We may use two approaches that are in fact equivalent. The first approach is presented below. It works in a way analogous to the computation of the Popov form we have presented. It just consider the fraction descriptions in a form that gain from the Popov form. Another point of view will be found in [40]; it is based on modules over a P.I.D and exploits the links, both theoretically and practically, between normal forms of matrix polynomials and normal form$s$ of matrices over a field under similarity. Computing the Hermite form of the Popov form $T(x)$ reduces to computing a polycyclic form [31] or shift-Hessenberg form [1] from the shift-form $A$ associated to $T(x)$ [40].

For the irreducible right fraction description with denominator $T(x)$ under Popov form, that we have computed at lemma 2 , one can readily construct a minimal realisation $\Sigma=(C, A, B)$ (as defined in $\S 2.1$ ). The matrix $A$ in $\mathcal{M}_{\nu, \nu}(K)$ can be chosen in shift form $[32,19]$ :

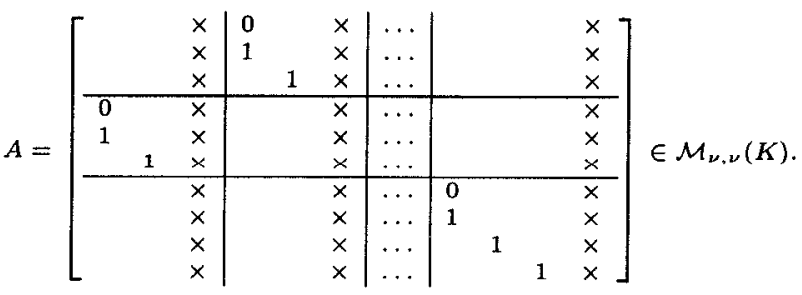

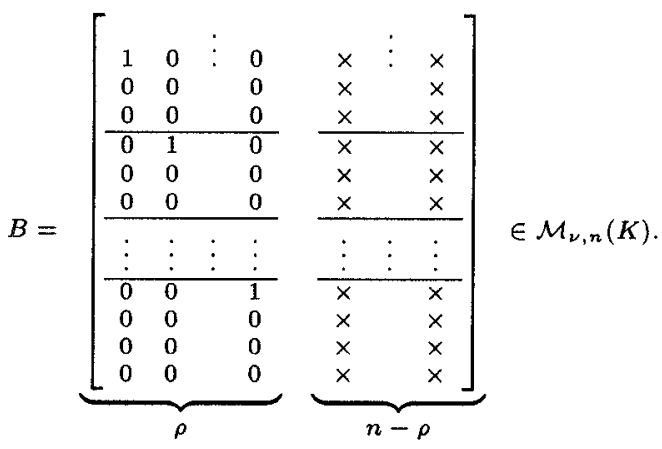

More precisely, $A$ and $B$ are obtained as follows. To construct $A$ we map $T(x)$ onto the above structure. Only the columns $j, 1 \leq j \leq \rho$ of $T(x)$ with nonzero column degree $d_{j}$ are relevant here. From the definition of the Popov form, in each row containing a zero degree pivot, all the other entries are zero, let us call them non relevant. Up to column and row permutations we may assume that the leading $\rho \times \rho$ submatrix of $T(x)$ gather together all the relevant entries of $T(x)$. Row operation are not allowed: they will be reflected back in the end matrix. The matrix $A$ is a $\rho \times \rho$ block matrix. The block $\left(r_{j}, j\right)$ is the companion block of minimal polynomial the pivot of the $j$-th column of $T(x)$. The other blocks $\left(r_{3}, l\right), l \neq j$, have nonzero entries only in their last column. This column is given by the coefficients of the corresponding entry $T_{r_{j}, l}(x)=\Sigma_{k=0}^{d_{j}-1}=t_{r_{j} l k} x^{k}$ of degree at most $d_{j}-1$ - requirement IV) of the Popov form - it is:

$$
{ }^{t}\left[-t_{r_{j} l 0},-t_{r_{j} l 1}, \ldots,-t_{r, l\left(d_{j}-1\right)}\right] .
$$

We omit the matrix $C$ since it is not involved in the computation. The matrix $B$ is an element of $\mathcal{M}_{\nu, n}(K)$. It is constructed as two blocks of columns vectors. The $\rho$ first column vectors of $B$ are canonical vectors of $K^{\nu}$. Let $\pi$ be the index permutation considered during the proof of proposition 1 such that the $r_{\pi(j)}$ are increasingly ordered and $d_{\pi(j)} \neq 0,1 \leq j \leq \rho$. The first column vector of $B$ is the first canonical vector and the $l$-th column vector of $B, 2 \leq l \leq \rho$, is the $\left(d_{\pi(1)}+\ldots+d_{\pi(l-1)}+1\right)$-th canonical vector of $K^{\nu}$. The $n-\rho$ last column vectors of $B$ are given by the columns with pivot equal to one. The entries of the $l$-th column vector of $B, \rho+1 \leq l \leq n$, are given by the coefficients of the entries of column $\pi(l)$ of $T(x)$, as done above at (12) for the nonpivot entries of the relevant part.

Now, we proceed as for the Popov form during the proof of proposition 1 since the realization and the transfer matrix satisfy identity (5). There are strong relationships between the entries of the Hermite normal form of the denominator matrix $T(x)$ and the columns of the block Krylov matrix $M(A, B, \nu)$ :

$$
\left[B_{1}, A B_{1}, \ldots, A^{\nu} B_{1}, \ldots, B_{n}, \ldots, A^{\nu} B_{n}\right] \in \mathcal{M}_{\nu, n \nu}
$$

where $B_{j}$ denotes the $j$-th column of $B$ for $1 \leq j \leq n$.

Lemma 3 The column Hermite normal form $R_{H}(x)$ of $T(x)$ in Popov form as given by lemma 2, can be computed in $O(\log (n d))$ solutions of $\mathcal{L}(2 n d)$.

Proof. We do not detail the proof, it is based on classical results in $[42,19]$. By construction (\$6.4.4, [19]),

$$
H(x)=C(x I-A)^{-1} B=\bar{N}(x) T^{-1}(x) .
$$

Since $(C, A, B)$ is a minimal realization of $H(x)$ then $[42,19]$ there exists a matrix $\Psi(x)$ in $\mathcal{M}_{\nu, n}(K[x])$ right coprime with 
$D(x)$ such that

$$
(x I-A)^{-1} B=\Psi(x) T^{-1}(x) .
$$

Now, $R_{H}(x)$ is right equivalent to $T(x)$ thus the same holds for it and for a matrix $\Phi(x)$ :

$$
(x I-A)^{-1} B=\Phi(x) R_{H}^{-1}(x) .
$$

We keep the same reasoning than for proposition 1 with $M(A, B, \nu)$ playing the role of matrix $M(\delta+1)$ (indeed, from a system-theoretic point of view they are both controllability matrices). We get that the entries of $R_{H}(x)$ are read-off the nullspace of $M(A, B, \nu)$. We may write a linear system which solutions gives the entries of $R_{H}(x)$. This linear system is computed by first isolating the $\nu$ lexicographically first independent columns of $M(A, B, \nu)$. Since the matrix is block Krylov, we can restrict ourselves to $\nu \times 2 \nu$ matrices as done in [25]. The cost is that of $O(\log (\nu))$ solutions of $\mathcal{L}(2 \nu)$. The fact that $2 \nu \leq 2 n d$ terminates the proof.

Algorithm 2. Computing the column Hermite form.

The Hermate form of $P(x)$ is computed from its Popov form $T(x)$ (algorithm 1). This is done by solving a block Krylov system (lemma 3). An equvvalent alternative method would be to compute a shiftHessenberg form of the constant matrix $A$ associated to $T(x)$.

Input: a polynomial matrux $P(x)$.

$T(x) \leftarrow$ the column Popov form $P(x)$.

$M(A, B, \nu) \leftarrow$ the block Krylov matrix (13).

$\bar{M}(A, B, \nu) \leftarrow$ the first indep. col. of $M(A, B, \nu)$.

$\mathcal{R}_{H} \leftarrow$ the solution of the corresponding linear system. Build $R_{H}(x)$ from the entries of $\mathcal{R}_{H}$.

Output: the column Hermate form $R_{H}(x)$ of $P(x)$.

\section{Cost analysis}

Using the results of previous sections we establish new cost bounds for the computation of the Hermite normal form of a matrix polynomial of dimension $n$ and of degree $d$. We let $n=O(\mu)$ and $d=O(\mu)$. For a survey of basic sequential and parallel complexities, e.g. for matrix or polynomial multiplication, we refer to [7].

Proposition 3 A reduction to the Hermite normal form for a non singular matrix in $\mathcal{M}_{n, n}(K[x])$ of degree $d$ can be computed in deterministic sequential time $\tilde{O}(n d M(n)+$ $M(n d))=O\left(\mu^{4.8}\right)$ over $K$ using fast arithmetic, or, $O^{-}\left(\mu^{6}\right)$ using standard arithmetic.

Proof. The first step of the solution consists in computing the Popov form of the matrix. By lemma 2, the corresponding cost is that of matrix polynomial inversion and of solution of $\mathcal{L}(n d)$ for a block Hankel matrix. Then, by lemma 3 , the Hermite form is obtained by performing $O(\log (n d))$ solutions of $\mathcal{L}(2 n d)$. During the first step, the inverted matrix is $n \times n$ thus the former cost is $O(M(n))$ operations on univariate polynomials of degree $O(n d)$. This gives $\tilde{O}(n d M(n))$ over $K$. For the second step, on can solve $\mathcal{L}(2 n d)$ in time $\tilde{O}(M(n d))$ using the algorihm in [25]. Thus the complexity given in [11] for matrix multiplication leads to the announced cost $O\left((n d)^{2.4}\right)$. Using standard arithmetic, unlike the eliminations in $[18,16]$ but as the probabilistic triangularization in [36], our algorithm is susceptable to a evaluation/interpolation scheme. Since two polynomials of degree $d$ are multiplied in $O\left(n^{2} d^{2}\right)$, the cost of matrix inversion is $O\left(n d \times n^{3}+n^{2} \times n^{2} d^{2}\right)=O\left(n^{4} d^{2}\right)$. The second step costs $O(\log (n d))$ times $O\left((n d)^{3}\right)$. We get $\bar{O}\left(n^{4} d^{2}+n^{3} d^{3}\right)=\bar{O}\left(\mu^{6}\right)$ for the whole computation. Clearly, the unique transformation matrix is obtained with the same bounds.

Proposition $4 A$ reduction to the Hermite normal form for a non singular matrix in $\mathcal{M}_{n, n}(K[x])$ of degree $d$ can be computed in $O\left(\log ^{4}(n d)\right)$ arithmetic parallel steps using $O\left(n^{3 / 2} d M(n)+(n d)^{1 / 2} M(n d)\right)=O\left(\mu^{5.8}\right)$ processors if $K$ is of characteristic zero. The computation can be performed in time $O\left(\log ^{3}(n d)\right)$ using $\left.O\left((n d)^{4} M(n d)\right)=O\left(\mu^{12}\right)^{8}\right)$ processors over an arbitrary field.

Proof. Over a field of characteristic zero, we refer to [33] for matrix inversion. The number of processors required is $O\left(n^{1 / 2} M(n)\right)$ for $O\left(\log ^{2} n\right)$ arithmetic steps. Together with the cost of the operations on polynomials of degree $n d$, in time $O(\log (n d))$ using $\tilde{O(n d)}$ processors, this gives $O\left(n^{3 / 2} d M(n)\right)$. Then, we follow results of [17] for the rank, of [22] for the nullspace $\left(O\left(\log ^{2} n\right)\right.$ steps $)$ and of [12] for the subset of independent columns $(\times O(\log n)$ steps), and we use, as proposed in [13], the parallel handling of [25] for the block Krylov system involved in lemma $3(\times O(\log n)$ steps). The Hermite form is computed from the Popov form in time $O\left(\log ^{4}(n d)\right)$ using $O\left((n d)^{1 / 2} M(n d)\right)$ processors.

Over an arbitrary field, the costs are dominated by the solution of $\mathcal{L}(2 n d)$. As for the processor bound of the solution of the nullspace problem in [9] this leads to nd times [7] the processor bound for the rank. This latter problem is solved using the algorithm in [30]. The number of steps if $O\left(\log ^{3}(n d)\right)$ using $O\left((n d)^{4} M(n d)\right)$ processors. The unique transformation matrix is obtained with the same bounds. $\square$

Comparing the sequential cost $O\left(\mu^{4.8}\right)$ of proposition 3 and the parallel work $O\left(\mu^{12.8}\right)$ of proposition 4 we see that our parallel solution is still far from processor-efficient. But no processor-efficient algorithms are known even for general linear systems solution, for rank and for maximal linearly independent subset over fields. However, processor-efficient Las Vegas algorithms are available in each case [22, 23, 12].

Proposition 5 There exists a Las Vegas type probabilistic algorithm to compute a reduction to the Hermite normal form of a non singular matrix in $\mathcal{M}_{n, n}(K[x])$ of degree $d$ running in $O\left(\log ^{4}(n d)\right)$ artthmetic parallel steps and

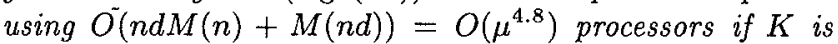
of characteristic zero or greater than $n d$. If $K$ is of positive characteristic $p$ lower than nd then the running time is $O\left(\log ^{5}(n d) / \log p\right)$.

Proof. We can use the same arguments than for the proof of proposition 4 but using the probabilistic versions of the algorithms. We refer to $[22,23]$ for the rank and the nullspace, to [12] for the independent subset and to $[25,13]$ to handle the block Krylov matrix.

\section{Conclusion}

We have given new complexity bounds for some operations on matrix polynomials. These have been obtained using a strong relationship (never exploited for practical purposes in matrix computer algebra) with linear system theory in control. Pursuing this approach, many technical work remains to be done. But the questions we did not tackle here (e.g the use of the Bezoutian, the boolean complexity, the case of singular matrices, links with Euclid's algorithm) should be solved as it has been done for scalar polynomials. 


\section{References}

[1] Augot, D., and Camion, P. The minimal polynomials, characteristic subspaces, normal bases and the Frobenius form. Tech. Rep. 2006, INRIA France, Aug. 1993.

[2] BARNETT, S. Greatest common divisor of two polynomials. Linear Algebra and its Applications 3 (1970), 7-9.

[3] BARnett, S. Greatest common divisor of several polynomials. Proc. Cambridge Philos. Society 70 (1971), 263-268.

[4] Barnett, S, and Lancaster, P. Some properties of the bezoutian for polynomial matrices. Linear Algebra and Multilinear Algebra 9 (1980), 99-110.

[5] Bely̆̌, V., Khazanov, V., and Kublanovskaya, V. Spectral problems for matrix pencils. Methods and algorithms III. Soviet J. Numer. Anal. Math. Model. 4, 1 (1989), 1951.

[6] Bini, D., and Gemignani, L. Fast parallel computation of the polynomial remainder sequence via Bezout and Hankel matrices. SIAM J. Comput. 24, 1 (Feb. 1995), 63-77.

[7] BINI, D., AND PAN, V. Polynomial and matrix computations. Birkhäuser, 1994

[8] Bitmead, R., Kung, S., Anderson, B., and Kailath, T. Greatest common divisors via generalized Sylvester and Bezout matrices. IEEE Trans. Automat. Control. 23, 6 (1978), 1043-1047

[9] Borodin, A., von zur Gathen, J, and Hopcroft, J. Fast parallel matrix and gcd computations. Information and Control 52 (1982), 241-256.

[10] Coppersmith, D. Solving homogeneous linear equations over $\mathrm{GF}(2)$ via block Wiedemann algorithm. Mathematics of Computation 62, 205 (1994), 333-350.

[11] Coppersmith, D., AND Winograd, S. Matrix multiplication via arithmetic progressions. In 19 th. Annual ACM Symp. Theory Comp. (1987), pp. 1-6.

[12] EberLy, W. Parallel independent subsets and matrix factorizations. In Proc. 3rd IEEE Conference on Parallel and Distributed Processing (1991), IEEE Computer Society Press.

[13] Gresbrecht, M. Nearly optimal algorithms for canonical matrix forms. SIAM Journal on Computing (1994). To appear.

[14] Gohberg, I., Lancaster, P., And Rodman, L. Matrix polynomials. Academic Press, New-York, 1982.

[15] GRAGG, W., AND LindQuist, A. On the partial realization problem. Linear Algebra and its Applications 50 (1983), 277-319.

[16] Hafner, J., AND MC CuRley, K. Asymptotically fast triangularization of matrices over rings. SIAM J. Comput. 20, 6 (1991), 1068-1083.

[17] Ibarra, O., Moran, S., ANd Rosier, L. A note on the parallel complexity of computing the rank of order $n$ matrices. Information Processing Letters 11 (1980), 162.

[18] Ilropoulos, C. Worst-case complexity bounds on algorithms for computing the canonical structure of finite abelian groups and the Hermite and Smith normal forms of an integer matrix. SIAM J. Comput. 18, 4 (1989), 658-669.

[19] KaILATh, T. Linear systems. Prentice Hall, 1980.

[20] Kalman, R., Falb, P., and Arbib, M. Topics in mathematical system theory. McGraw-Hill, New-York, 1969.

[21] Kaltofen, E., Krishnamoorthy, M., and Saunders, B. Fast parallel computation of Hermite and Smith forms of polynomials matrices. SIAM J. Alg. Disc. Meth. 8 4, pp 683-690 (1987).

[22] Kaltofen, E., AND PAN, V. Processor efficient parallel solution of linear systems over an abstract field. In Proc. 3rd Annual ACM Symposium on Parallel Algorithms and Architecture (1991), ACM-Press.
[23] Kaltofen, E., AND PAN, V. Processor efficient parallel solution of linear systems II: the general case. In Proc. 33rd IEEE Symp. Foundations of Computer Science, Pittsburg, USA (1992).

[24] Karcanias, N., ANd Mitrouli, M. A matrix pencil based numerical method for the computation of the gcd of polynomials. IEEE Trans. Autom. Contr. 39, 5 (1994), 977-981.

[25] Keller-Gehrig, W. Fast algorithms for the characteristic polynomial. Theoretical Computer Science 36 (1985), 309317.

[26] KUNG, S. Multivariable and multidimensional systems: analysis and design. PhD thesis, Dpt. of Electrical Engineering, Stanford University, June 1977.

[27] Labhalla, S., Lombardi, H., and Marlin, R. Algorithmes de calcul de la réduction d'Hermite d'une matrice à coefficients polynomiaux. Theoretical Computer Science (1995). To appear.

[28] MACDuffeE, C. The theory of matrices. Chelsea, New-York, 1956.

[29] Manocha, D., AND CANnY, J. Multipolynomial resultants and linear algebra. In International Symposium on Symbolic and Algebraic Computation, Berkeley California USA (July 1992), ACM Press.

[30] Mulmuley, K. A fast parallel algorithm to compute the rank of a matrix over an arbitrary field. Combinatorica 7, 1 (1987), 101-104.

[31] OzeLlo, P. Calcul exact des formes de Jordan et de Frobenius d'une matrice. $\mathrm{PhD}$ thesis, Université Scientifique et Médicale de Grenoble, France, 1987.

[32] Popov, V. Invariant description of linear, time-invariant controllable systems. SIAM J. Contr. 10 (May 1972), 252264.

[33] Preparata, F., and Sarwate, D. An improved parallel processor bound in fast matrix inversion. Inform. Proc. Lett. 7 (1978), 148-150.

[34] Pták, V., AND Wimmer, H. On the Bezoutian for polynomial matrices. Linear Algebra and its Applications 71 (1985), $267-272$.

[35] QuÉRÉ, M., AND VILlaRd, G. Matrices quasi-Hankel et Bezoutiennes pour le calcul de la forme normale d'Hermite de matrices polynomiales. Preprint IMAG, Grenoble France, 1996.

[36] Storjohann, A. Computation of Hermite and Smith normal forms of matrices. Master's thesis, 1994. University of Waterloo, Canada.

[37] Storjohann, A., ANd Labahn, G. Asymptotically fast computation of Hermite normal forms of integer matrices. In International Symposium on Symbolic and Algebraic Computation, Zurich, Suisse (July 1996), ACM Press.

[38] VAN DOOREN, P., AND DEWILDE, P. The eigenstructure of an arbitrary polynomial matrix: computational aspects. Linear Algebra and its Applications 50 (1983), 545-579.

[39] Van Dooren, P., Dewilde, P., and Vandewalle, J. On the determination of the Smith-Macmillan form of a rational matrix from its Laurent expansion. IEEE Trans. Cir. Sys. 26, 3 (March 1979), 180-189.

[40] VILLARD, G. Some algorithms for matrix polynomials. Tech. Rep. RT157, IMAG Grenoble France, 1996.

[41] Wolovich, W. Linear multivariable systems. SpringerVerlag, New-York, 1974

[42] WolOVICH, W., AND FALB, P. On the structure of multivariable systems. SIAM J. Control AC-12, 3 (1969), 437-451.

[43] YovlA, D , ANd Tissi, P. N-port synthesis via reactance extraction, part I. IEEE Int. Conv. Rec. 14 (1966), 183205. 
G VILLARD is Chargé de Recherche at the Centre National de la Recherche Scientifique since 1990. Dr. Villard obtained his Ph.D. in 1988 (University of Grenoble - F). He is a member of IMAG - Informatique et Mathématiques Appliquées de Grenoble and his research interests concern parallel computer algebra and linear algebra for differential equations. 